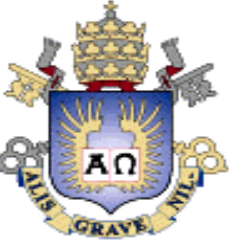

Marco Aurélio Simão Freire

Distribuições de Retornos, Volatilidades e Correlações no Mercado Acionário Brasileiro

Dissertação apresentada como requisito parcial para obtenção do título de Mestre pelo Programa de PósGraduação em Economia da PUC-Rio.

Orientador: Marcelo Cunha Medeiros 
Marco Aurélio Simão Freire

\section{Distribuições de Retornos, Volatilidades e Correlações no Mercado Acionário Brasileiro}

Dissertação apresentada como requisito parcial para obtenção do título de Mestre pelo Programa de PósGraduação em Economia da PUC-Rio. Aprovada pela Comissão Examinadora abaixo assinada.

Marcelo Cunha Medeiros

Orientador

Marcelo Fernandes

FGV-RJ

Álvaro de Lima Veiga Filho

PUC-RJ

Cristiano Augusto Coelho Fernandes

PUC-RJ

Gisele Guimarães Cittadino

Coordenador(a) Setorial do Centro de Ciências Sociais - PUC-Rio

Rio de Janeiro, 13 de abril de 2004 
Todos os direitos reservados. É proibida a reprodução total ou parcial do trabalho sem autorização da universidade, do autor e do orientador.

\section{Marco Aurélio Simão Freire}

Graduou-se em Economia na PUC-Rio em 2001, tendo logo em seguida ingressado no Mestrado em Economia da mesma instituição. Tem como área de pesquisa Econometria e Finanças, onde desenvolveu sua monografia e sua tese. Atualmente trabalha no Bank Boston como gestor de um fundo multimercados.

Ficha Catalográfica

Freire, Marco Aurélio Simão

Distribuições de retornos, volatilidades e correlações no mercado acionário brasileiro / Marco Aurélio Simão Freire ; orientador: Marcelo Cunha Medeiros. - Rio de Janeiro : PUC, Departamento de Economia, 2004.

73 f. : il. ; $30 \mathrm{~cm}$

Dissertação (mestrado) - Pontifícia Universidade Católica do Rio de Janeiro, Departamento de Economia.

Incluí referências bibliográficas.

1. Economia - Teses. 2. Volatilidade realizada. 3. Dados de alta frequência. 4. Modelos GARCH multivariados. 5. Distribuições de retornos. 6. Análise de risco. I. Medeiros, Marcelo Cunha. II. Pontifícia Universidade Católica do Rio de Janeiro. Departamento de Economia. III. Título. 


\section{Agradecimentos}

À minha noiva Caroline Botelho Machado por todo companheirismo, compreensão e amor ao longo dos últimos anos.

À minha avó que sempre me incentivou em meus estudos.

À minha mãe pelas importantes contribuições e palavras de apoio.

Ao meu orientador Professor Marcelo Medeiros por toda atenção e suporte que foram além do ambiente acadêmico.

À CAPES pelo auxílio concedido sem o qual este trabalho não poderia ter sido realizado. 


\section{Resumo}

Freire, Marco Aurélio Simão. Distribuições de Retornos, Volatilidades e Correlações no Mercado Acionário Brasileiro. Rio de Janeiro, 2004. 73p. Dissertação de Mestrado - Departamento de Economia, Pontifícia Universidade Católica do Rio de Janeiro.

A hipótese de normalidade é comumente utilizada na área de análise de risco para descrever as distribuições dos retornos padronizados pelas volatilidades. No entanto, utilizando cinco dos ativos mais líquidos na Bovespa, este trabalho mostra que tal hipótese não é compatível com medidas de volatilidades estimadas pela metodologia EWMA ou modelos GARCH. Em contraposição, ao extrair a informação contida em cotações intradiárias, a "metodologia de volatilidade realizada" origina retornos padronizados normais, potencializando ganhos no cálculo de medidas de Valor em Risco. Além disso, são caracterizadas as distribuições de volatilidades e correlações de ativos brasileiros e, em especial, mostra-se que as distribuições das volatilidades são aproximadamente lognormais, enquanto as distribuições das correlações são aproximadamente normais. A análise é feita tanto de um ponto de vista univariado quanto multivariado e fornece subsídio para a melhor modelagem de variâncias e correlações em um contexto de grande dimensionalidade.

\section{Palavras-chave}

Volatilidade realizada; dados de alta freqüência; modelos GARCH multivariados; distribuições de retornos; análise de risco. 


\section{Abstract}

Freire, Marco Aurélio Simão. Distributions of Returns, Volatilities and Correlations in the Brazilian Stock Market. Rio de Janeiro, 2004. 73p. Msc.Dissertation - Departamento de Economia, Pontifícia Universidade Católica do Rio de Janeiro.

The normality assumption is commonly used in the risk management area to describe the distributions of returns standardized by volatilities. However, using five of the most actively traded stocks in Bovespa, this paper shows that this assumption is not compatible with volatilities estimated by EWMA or GARCH models. In sharp contrast, when we use the information contained in high frequency data to construct the realized volatilies measures, we attain the normality of the standardized returns, giving promise of improvements in Value at Risk statistics. We also describe the distributions of volatilities and correlations of the brazilian stocks, showing that the distributions of volatilities are nearly lognormal and the distribuitions of correlations are nearly Gaussian. All analysis is traced both in a univariate and a multivariate framework and provides background for improved high-dimensional volatility and correlation modelling in the brazilian stock market.

\section{Keywords}

Realized volatility; high frequency data; multivariate GARCH models; return distributions; risk management. 


\section{Sumário}

1. Introdução

2. Revisão Teórica: Métodos de volatilidades e covariâncias

2.1. Metodologia do Riskmetrics (J.P. Morgan (1997)) 15

2.2. Modelos da Família GARCH 17

2.2.1. Constant Correllation GARCH (Bollerslev (1990)) 20

2.2.2. GARCH Ortogonal (Alexander (2000)) 21

2.3. Volatilidade Realizada (Anderson et al. (2003)) 23

3. Dados e a construção das medidas de volatilidade e covariâncias 28

4. Distribuições univariadas e multivariadas de retornos e volatilidades 35

4.1. Distribuições Condicionais Univariadas de Retornos 35

4.2. Distribuições Condicionais Multivariadas de Retornos 40

4.3. Distribuições Univariadas de Volatilidades e Correlações 41

4.3.1. Distribuições de Volatilidades 42

4.3.2. Distribuições de Correlações 47

4.4. Distribuições Multivariadas de Volatilidades e Correlações 51

5. Experimentos de Valor em Risco 53

5.1. Conceito de Valor em Risco 53

5.2. Análise dentro da amostra 55

5.2.1. Testes de Cobertura Incondicional e Condicional 55

5.2.2. Teste da Transformação Normal de Berkowitz (2001) 58

5.2.3.Modelos de Valor em Risco e Exigência de Capital 60

5.3. Análise dentro da amostra 63

6. Conclusões 68

$\begin{array}{ll}\text { 7. Referências Bibliográficas } & 70\end{array}$ 


\section{Lista de Figuras}

Figura 1- Retornos diários de Petrobrás, Embratel, Bradesco, Telemar 28 e Vale do Rio Doce

Figura 2- Volatilidades diárias realizadas de Petrobrás, Embratel, 32 Telemar, Bradesco e Vale do Rio Doce

Figura 3- Volatilidades diárias estimadas por EWMA de Petrobrás, 32 Embratel, Telemar, Bradesco e Vale do Rio Doce

Figura 4- Correlações diárias realizadas de Petrobrás, Embratel, 33 Telemar, Bradesco e Vale do Rio Doce

Figura 5- Correlações diárias estimadas por EWMA de Petrobrás, 34 Embratel, Telemar, Bradesco e Vale do Rio Doce

Figura 6- Estimação não paramétrica das densidades dos retornos 39 padronizados pelas medidas de volatilidade realizada

Figura 7- Estimação não paramétrica das densidades de volatilidades 45 e logarítimo das volatilidades realizadas

Figura 8- Autocorrelações amostrais dos logarítimos das volatilidades 46 realizadas

Figura 9- Estimação não paramétrica das densidades das correlações 49 diárias estimadas da Telemar

Figura 10- Autocorrelações amostrais das correlações diárias 50 realizadas

Figura 11- Intervalos de confiança de 99\% para os retornos diários da 63 Petrobrás (dentro da amostra)

Figura 12- Intervalos de confiança de 99\% para os retornos diários da 67 Petrobrás (fora da amostra) 


\section{Lista de Tabelas}

Tabela 1- Análise de viés do estimador de volatilidade realizada $\quad 30$

Tabela 2- Análise de eficiência do estimador de volatilidade realizada 31

Tabela 3- Distribuições dos retornos diários dos ativos - retornos 35 incondicionais

Tabela 4- Distribuições dos retornos diários dos ativos - retornos 37 padronizados (EWMA)

Tabela 5- Distribuições dos retornos diários dos ativos - retornos 37 padronizados (GARCH)

Tabela 6- Distribuições dos retornos diários dos ativos - retornos 37 padronizados (EGARCH)

Tabela 7- Distribuições dos retornos diários dos ativos - retornos 38 padronizados (volatilidade realizada)

Tabela 8- Testes da hipótese de Normalidade dos retornos 41 Multivariados

Tabela 9- Distribuições das Volatilidades diárias - desvio padrão

Tabela 10- Distribuições das Volatilidades diárias - log desvio padrão 43

Tabela 11- Distribuições covariâncias/ correlações - covariâncias 47

Tabela 12- Distribuições covariâncias/ correlações - correlações 47

Tabela 13- Distribuições multivariadas de volatilidades 51

Tabela 14- Percentual de exceções Valor em Risco 56

Tabela 15- Teste de cobertura incondicional 56

Tabela 16- Teste de cobertura condicional 58

Tabela 17- Teste da transformação normal 60

Tabela 18- Análise de exigência de capital 62

Tabela 19- Percentual de exceções Valor em Risco (fora da amostra) 64

Tabela 20- Teste de cobertura incondicional (fora da amostra) 65

Tabela 21- Teste de cobertura condicional (fora da amostra) 65

Tabela 22- Teste da transformação normal (fora da amostra) 65

Tabela 23- Análise de exigência de capital (fora da amostra) 66 